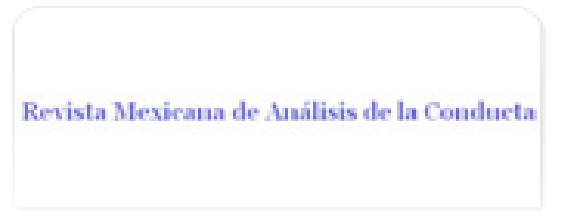

Revista Mexicana de Análisis de la Conducta ISSN: 0185-4534

editora@rmac-mx.org

Sociedad Mexicana de Análisis de la Conducta México

López Corral, Esther; Santoyo Velasco, Carlos

Asimetría de la interacción conflictiva de cónyuges violentos: la prueba de un modelo Revista Mexicana de Análisis de la Conducta, vol. 30, núm. 2, diciembre, 2004, pp. 115-138

Sociedad Mexicana de Análisis de la Conducta

Guadalajara, México

Disponible en: http://www.redalyc.org/articulo.oa? $\mathrm{id}=59330201$

- Cómo citar el artículo

- Número completo

- Más información del artículo

- Página de la revista en redalyc.org

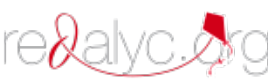

Sistema de Información Científica

Red de Revistas Científicas de América Latina, el Caribe, España y Portugal Proyecto académico sin fines de lucro, desarrollado bajo la iniciativa de acceso abierto 


\title{
ASIMETRÍA DE LA INTERACCIÓN CONFLICTIVA DE CÓNYUGES VIOLENTOS: LA PRUEBA DE UN MODELO
}

\author{
ASYMMETRY OF THE CONFLICTING INTERACTION OF \\ VIOLENT SPOUSES: THE TEST OF A MODEL \\ ESTHER LÓPEZ CORRAL Y CARLOS SANTOYO VELASCO \\ ESCUELA LIBRE DE PSICOLOGÍA, A.C.-UNIVERSIDAD PEDAGÓGICA \\ NACIONAL Y FACULTAD DE PSICOLOGÍA, UNIVERSIDAD NACIONAL \\ AUTÓNOMA DE MÉXICO.
}

\begin{abstract}
RESUMEN
Con base en el modelo teórico de la proporcionalidad de la injusticia, derivado de la teoría de integración de la información, se describen interacciones diádicas de cónyuges violentos comparados con cónyuges no violentos. Participaron 22 parejas de cónyuges (11 violentos y 11 de control) quienes discutieron 3 temas en sesión videograbada: eventos cotidianos, resolución de conflicto y tópico placentero. Observadores entrenados registraron la discusión utilizando el Sistema Observacional de la Interacción Conyugal, cuyas categorías se agruparon en Contribuciones y Resultados de la relación con valencia positiva y negativa. Análisis secuenciales con retardo proporcionaron los datos para operar el modelo. Se encontró mayor asimetría para cónyuges violentos que de control, en intercambios positivos y negativos. Análisis secuenciales sin retardo indican mayor sincronía para cónyuges violentos y de control en intercambios negativos y positivos, respectivamente. Se analiza el patrón excitatorio e inhibitorio conductual de 2 díadas. Se discute la generali-
\end{abstract}

1. Agradecemos el apoyo recibido por parte del Consejo Nacional de Ciencia y Tecnología (40442-H) y por los Servicios Educativos del Estado de Chihuahua, en la realización de esta investigación. El presente estudio fue conducido como parte de la investigación para la tesis doctoral de Esther López Corral, bajo la dirección del Dr. Carlos Santoyo Velasco. Cualquier comunicación dirigirse a esther_lopez_corral@yahoo.comy/o carsan@servidor.unam.mx 
dad del modelo al fundamentarlo en la observación de patrones de conducta como una herramienta útil a la investigación.

Palabras clave: interacción marital, injusticia, asimetría, observación, cónyuges violentos.

\section{SUMMARY}

Based on the theoretical model of the proportionality of the injustice, derived from the integration information theory, dyadic interactions of violent partners compared with non-violent partners are described. Twenty-two pairs of partners participated (11 violent and 11 controls), who discussed three topics in videotaped sessions: daily events, resolution of conflict and, pleasant topic. Trained observers registered videotapes using the Conyugal Interaction Observational System, whose categories were grouped into Inputs and Outputs of the relationship with positive and negative valence. Sequential analyses with $\operatorname{Lag}=1$, provided the data to operate the model. Greater asymmetry for violent partners than for controls was founded in positive and negative exchanges. Sequential analyses with $\mathrm{Lag}=0$ indicate greater synchrony for violent partners and for controls in negative and positive exchanges, respectively. The excitatory and inhibitory behavioral pattern for 2 dyads is analyzed. The generality of the model based on the observation of behavioral patterns is discussed as an useful research tool.

Key words: marital interaction, unfairness, asymmetry, observation, violent partners.

Para el estudio de los intercambios diádicos, modelos como la teoría de la equidad de Adams (1965) y la Teoría de Integración de la Injusticia (Farkas, 1991; ver también Anderson, 1991) parten del supuesto de que los actores implicados invierten costos, esfuerzos o atributos para que el intercambio se verifique. Si cualquier parte involucrada en el intercambio reconoce la existencia de un atributo en el otro o en ellos mismos, entonces el atributo se constituye en una Contribución. Por otra parte, estos modelos consideran que en cualquier interacción humana, se esperan retribuciones justas a las contribuciones emitidas por una de las partes, lo cual se define como Resultados (Adams, 1965). La teoría de Integración de la Injusticia (Farkas, 1991, p.54) se resume en la siguiente ecuación:

$\mathrm{la}=\mathrm{Ca} /(\mathrm{Ca}+\mathrm{Cb})-\mathrm{Ra} /(\mathrm{Ra}+\mathrm{Rb})$

En la que,

la= denota injusticia a la persona $\underline{A}$. 
$\mathrm{C}=$ contribuciones de la persona $\underline{a}$ y $\underline{b}$, respectivamente.

$\mathrm{R}=$ resultados para la persona $\underline{a} \underline{\mathrm{y}} \underline{\mathrm{b}}$, respectivamente

Este modelo de injusticia incluye tres procesos de comparación. Una comparación interpersonal, para determinar la contribución relativa, otra comparación interpersonal similar, para determinar el resultado relativo, y la tercer comparación se realiza con ambas razones relativas para determinar la injusticia (Farkas, 1991).

La peculiaridad conceptual de este modelo, a diferencia de otros modelos previos, estriba en que la comparación final se realiza entre contribuciones o resultados relativos, es decir, la contribución del sujeto a se considera en función de lo que ambos participantes contribuyen a la relación de manera conjunta, y ello es comparado con el resultado que obtiene el sujeto $\underline{a}$, en función de lo que ambos obtienen de la relación de manera conjunta. Esta formulación diádica implica información equivalente de ambos participantes en cada elemento de la ecuación.

El carácter integrativo del modelo se satisface en dos sentidos. En primer lugar, se integra información de dos ámbitos diferentes: contribuciones y resultados; en segundo lugar, se involucra información de dos fuentes distintas que corresponden a los participantes que realizan la comparación. En ambos sentidos, se integra información de dos dominios distintos para producir un tercer elemento que resume o sintetiza a los anteriores.

Es de suponerse que entre mayor diferencia exista entre ambas razones relativas, se reportará mayor injusticia. De manera complementaria, cuando las diferencias se aproximan a cero, menor injusticia se experimentará.

Por otra parte, Adams (1965) plantea que tanto la cualidad de las contribuciones, como la valencia positiva o negativa de los resultados, son determinantes cruciales en el intercambio social. Al incorporar esta distinción en el modelo, se derivan dos versiones de la ecuación original:

y

$$
l a=C p a /(C p a+C p b)-R p a /(R p a+R p b)
$$

$l a=C n a /(C n a+C n b)-R n a /(R n a+R n b)$

En las que,

la= denota injusticia a la persona $\underline{A}$.

$\mathrm{Cp}=$ contribuciones positivas de la persona $\underline{\mathrm{a}}$ y $\underline{\mathrm{b}}$, respectivamente

$\mathrm{Rp}=$ resultados positivos para la persona $\underline{\mathrm{a}}$ y $\underline{\mathrm{b}}$, respectivamente

$\mathrm{Cn}=$ contribuciones negativas de la persona $\underline{\mathrm{a}} \mathrm{y} \underline{\mathrm{b}}$, respectivamente

$\mathrm{Rn}=$ resultados negativos para la persona $\underline{a}$ y $\underline{b}$, respectivamente. 
Así, la ecuación 2, refiere un modelo de comparación social que incorpora contribuciones y resultados con valencia positiva, mientras que la ecuación 3 , plantea el mismo modelo pero con valencia negativa en sus elementos, en lo subsiguiente designados como modelo positivo y negativo, respectivamente.

La teoría de integración de la injusticia, se ha utilizado en diferentes escenarios y los resultados proporcionan buen soporte para ella, pero pocos de estos estudios se han conducido en el área conyugal. La investigación en el área marital es un campo fértil y atractivo a la aplicación de dicho modelo, por ser un ámbito en que comparaciones interpersonales se realizan de manera cotidiana y frecuentemente constituyen procesos desapercibidos por los participantes o por terceras personas.

La literatura refiere algunos estudios que vinculan la equidad con la violencia conyugal, pero dichos estudios son conducidos al margen del modelo teórico. Por ejemplo, Vivian y Langhinrichsen-Rohling (1994) reportan que ambos factores están relacionados, entendiendo equidad como victimización mutua. Leonard y Senchak (1996) reportan que la iniquidad percibida de poder, entre otros constructos, estuvo relacionada prospectivamente con la agresión del esposo. Patterson (1982) refiere que existe una relación entre conducta inadaptada y desequilibrio en los intercambios familiares.

Sin embargo, investigaciones realizadas que estudian estos modelos de comparación social, presentan algunos inconvenientes metodológicos, ya que confían de manera exagerada en información obtenida de medidas indirectas de los procesos bajo estudio, tales como el autorreporte, el juicio subjetivo del participante, o información retrospectiva, característica compartida con la mayor parte de las investigaciones en el área marital. Esta peculiaridad es señalada por la literatura científica como un grave problema en la investigación del área, ya que el extendido uso de medidas de autorreporte y de personalidad, implica que buena parte de las conclusiones que se dan por ciertas, se derivaron de fuentes de información imprecisa. Además, Patterson (1982) afirma que es necesario conducir estudios que no confíen exclusivamente en datos de autorreporte.

Por otra parte, se ha señalado que el campo de investigación marital se encuentra plagado con métodos de varianza común, y se distingue este método, como un grave defecto en la investigación marital, que consiste en usar el mismo método de medición para medir dos diferentes ideas o constructos, lo cual produce más altas correlaciones, que midiendo los constructos con métodos diferentes. Además, se reconoce que los mejores predictores de conducta potencial se basan en datos de conducta previa en similares situaciones (Gottman, 1998).

La investigación actual en el área ha progresado del uso de medidas de autorreporte y de la personalidad, a aproximaciones multimétodo que involucran el estudio de interrelaciones entre conducta interaccional, percepción y fisiología (Gottman \& Notarius, 2002). 
Así mismo, gran parte de los estudios son conducidos desde una perspectiva individualista, ya que manejan información solamente de uno de los cónyuges. Por ello se considera que existe una sobrerrepresentatividad femenina en estudios de violencia (Widom, 1989; Wilson \& Herrnstein, 1985). A diferencia de estos, entre los estudios que manejan una perspectiva diádica pueden señalarse los de Cascardi y Vivian (1995), Holtzworth-Munroe, Markman, O'Leary y Neidig (1995), Langhinrichsen-Rohling, Neidig y Thorn (1995), Margolin (1987) y O'Leary, Malone y Tyree (1994).

La discusión anterior refleja la necesidad de trascender los obstáculos y deficiencias señalados en la literatura, con una estrategia apropiada que satisfaga los inconvenientes que enfrenta la investigación en el área.

Abordar el estudio a través de la utilización de medidas directas observacionales con una perspectiva diádica, constituye una manera más válida y confiable de estudiar la interacción conyugal, especialmente la conflictiva y, al mismo tiempo, proporciona un panorama más completo del que ofrece una perspectiva individualista.

Al emplear el modelo teórico de integración de la injusticia con datos derivados de los intercambios sociales conyugales y representarlos gráficamente, es posible establecer una relación de simetría/asimetría entre ambos elementos del modelo. Los conceptos de simetría/asimetría (Gottman, 1979), reciprocidad y sincronía (Patterson, 1982), se han estudiado previamente en la investigación conductual mostrando su poder descriptivo y de predicción.

Desde la perspectiva diádica y conductual, se considera que ambos participantes en un contexto violento tienden a desempeñar un papel recíproco, envolviéndose en episodios cada vez más violentos, severos e intensos (Cascardi \& Vivian, 1995; Potegal \& Knutson, 1994). Existe buen soporte para la idea de que la violencia marido-esposa yace sobre un continuum de severidad (Sugarman, Aldarongo \& Boney, 1996), y que la coerción física en relaciones de parejas de novios es recíproca (Potegal \& Knutson, 1994). La hipótesis que subyace a estos estudios establece que la coerción produce violencia (Patterson, 1982).

Además, Babcock, Waltz, Jacobson y Gottman (1993) señalan que la violencia puede desempeñar un papel funcional para el individuo, al ser una conducta compensatoria para ocultar la falta de control conductual de los maridos en otras áreas del matrimonio. Con ellos coinciden Wilson y Herrnstein (1985) quienes afirman que la violencia puede ser una forma para restablecer la equidad, por lo que es necesario considerar el sentido de equidad o de justicia del individuo que realiza las comparaciones, partiendo del supuesto de que la conducta está determinada por sus consecuencias y que la relación entre costos y beneficios de cometer actos violentos y de no cometerlos, son determinantes cruciales en la violencia conyugal.

A partir de esto, se espera que el modelo teórico asumido, logre dife- 
renciar patrones conductuales de cónyuges violentos al compararlos con cónyuges de control, y que tales diferencias conductuales se relacionen con la simetría/asimetría establecida en la relación. Se hipotetiza que cónyuges violentos mostrarán más asimetría que los de control en sus comparaciones interpersonales. Por otra parte, se espera que cónyuges violentos demuestren mayores niveles de reciprocidad en aquellas conductas que favorecen el conflicto de la relación, a diferencia de los controles.

La simetría/asimetría de una relación puede verificarse estadísticamente mediante análisis de regresión. Una relación equitativa o simétrica se establece cuando se presentan valores similares y se producen diferencias de cero al comparar ambas razones del modelo. La simetría ideal sería establecida, al presentarse valores relativos de .5 en ambos ejes.

De acuerdo al modelo, relaciones asimétricas se establecerían al ser diferentes ambas razones, y por lo tanto, producirse diferencias tanto positivas como negativas. Al representar gráficamente este tipo de relaciones, los puntos se alejarían del .5 en ambos ejes y el análisis de regresión indicaría valores del intercepto cercanos a cero y valores de la pendiente cercanos a uno.

De esta manera, se propone que el objetivo de este trabajo sea extender y evaluar el modelo teórico de integración de la injusticia, al estudio de la interacción conyugal conflictiva desde una perspectiva conductual. Con lo que se pretende demostrar que el modelo es útil para describir, diferenciar y predecir relaciones maritales actuales, extendiendo la generalidad y alcance explicativo del mismo, sobre aquellos modelos basados en medidas indirectas como fuente de información primordial.

Al mismo tiempo, se evalúa la capacidad predictiva de los modelos propuestos, para describir las relaciones de simería/asimetría de contribuciones y resultados derivados de diferentes situaciones de discusión entre díadas de cónyuges violentos comparados con parejas control acopladas. Para ello se utilizó la observación conductual de los episodios sociales bajo diferentes contextos de relación.

De manera complementaria, se hace necesario analizar la contribución diferencial de los cónyuges en relaciones conflictivas, y diferenciar mecanismos de control mutuo que contribuyen o son incompatibles al desarrollo de patrones de violencia.

\section{MÉTODO}

\section{Participantes}

Participaron en el estudio 22 parejas de cónyuges $(\underline{N}=44)$ de la ciudad de Chihuahua, obtenidas a partir de un muestreo no probabilístico. La mitad 
de la muestra constituyó el grupo violento, formado por 11 parejas de cónyuges $(\underline{n}=22)$ con antecedentes de violencia conyugal, contactados a través de registros oficiales. La mitad restante 11 parejas de cónyuges, conformó el grupo de control, quienes fueron acoplados con el grupo violento en las variables de edad, ingreso económico mensual, hijos, empleo de la esposa y duración del matrimonio (Stroebe, Stroebe, Abakoumkin \& Schut, 1996), quienes aceptaron participar voluntariamente.

La muestra total presentó un promedio de edad de 35.07 años con una desviación estándar de 10.87 años; y un promedio de ingreso económico mensual de 3,932 pesos con una desviación estándar de 3,253 pesos. Presentaron un promedio de 9.1 años de vivir en pareja con una desviación estándar de 8.7 años. El 52.3\% indicó que vivía en su primer matrimonio, mientras que el $38.6 \%$ reportó vivir sus segundas nupcias. El grupo de cónyuges violentos debió reunir el requisito de presentar por lo menos un reporte de violencia conyugal ante la policía o un ministerio público, en el año anterior a la fecha de contacto (Anglin \& Holtzworth-Munroe, 1997).

Materiales y Aparatos.

Se utilizó el Sistema Observacional de la Interacción Conyugal (SOIC) derivado a partir de estudios previos (López \& Santoyo, 2001). El SOIC consiste en 13 categorías conductuales (ver Apéndice 1) denominadas Verbalización acerca del Tema (V), Habla Simultánea (Hs), Aprobación (A), Emisión (Emis), Desacuerdo (D), Defensa (Df), Provocación (P), Dominante (Do), Otras Conductas $(\mathrm{O})$, Interacción Física $(\mathrm{F})$, Humor $(\mathrm{H})$, Contacto Visual $(\mathrm{CV})$ y Pérdida de Contacto Visual (X).

Para la utilización del sistema se diseñó un formato de registro que define un Registro Activado por Unidad de Tiempo (RAUT) de 10 segundos (Quera, 1991), con el que se registra la conducta de maridos y esposas, e incluye información necesaria para facilitar la identificación posterior del registro. Los observadores fueron dos estudiantes de licenciatura entrenados, quienes cumplieron con el requisito de obtener cinco sesiones consecutivas, con un índice de concordancia interobservador mayor a .85 .

Se utilizaron dos cámaras de video, videocintas para la grabación, fotocopias del formato de registro, reproductoras de video, cronómetros, dos tarjetas que indicaban los temas que debían abordar en la discusión y una oficina libre de ruidos extremos para llevar a cabo la grabación.

\section{Procedimiento}

Primeramente se realizó el contacto en las oficinas de instituciones encargadas de atender problemas de violencia familiar (Ehrensaft \& Vivian, 1996), 
y se obtuvo la información de varias parejas de cónyuges que acudieron a dichas oficinas durante el año previo, para reportar algún incidente de violencia conyugal, se excluyeron los casos de violencia sexual. Posteriormente se realizaron visitas domiciliarias para invitar a los cónyuges a participar en un estudio sobre la "Calidad de Vida Marital", asegurando la estricta confidencialidad de la información que proporcionaran, y ofreciendo gratificar económicamente con 500 pesos su completa participación en el estudio, como una estrategia válida para incrementar la motivación de las parejas a participar (ver Babcock et al., 1993; Carstensen, Gottman \& Levenson, 1995; Heyman, O'Leary \& Jouriles, 1995; Levenson, Carstensen \& Gottman, 1994).

Del total de las parejas contactadas de esta manera, solamente cuatro de ellas aceptaron participar, lo que representa un $12.5 \%$ en la tasa de participación. El resto de participantes fueron parejas que voluntariamente aceptaron participar en el estudio, quienes también reunieron el requisito de presentar por lo menos un reporte policiaco de violencia conyugal.

Se solicitó a los participantes que acudieran en una sola ocasión a una cita a la institución afiliante del primer autor, y que realizaran una discusión sobre tres temas: a) Eventos Cotidianos b) Resolución de un Conflicto, y c) Tópico Placentero (Gottman, 1993). Aquellas parejas que aceptaron participar y acudieron a la cita, se condujeron a la oficina destinada para realizar su plática y ésta fue videograbada. La investigadora responsable indicó a los sujetos cada 20 minutos, que cambiaran de tema. Posteriormente las cintas fueron retiradas y las parejas de cónyuges conducidas a otra oficina para realizar una entrevista. Los resultados de esta entrevista no serán reportados en este trabajo.

Las videocintas fueron registradas por los observadores entrenados y se determinó la calidad de los datos observacionales a través del Índice de Concordancia Interobservador e Índice de Kappa de Cohen, que incluye una estimación de la probabilidad de acuerdos debidos al azar.

Todas las observaciones presentaron índices de concordancia interobservador superiores a .85 e índices de Kappa de Cohen superiores a .7, por lo cual los datos empleados en esta investigación, de acuerdo con el criterio referido por Bakeman y Gottman (1986/1989), son de una excelente calidad.

Todas las categorías originales del sistema fueron recategorizadas en Contribuciones y Resultados. Ya que una contribución es cualquier atributo del individuo o de su contraparte y que un resultado es cualquier retribución hecha a las contribuciones del otro, se consideraron como contribuciones aquellas conductas que presentó un miembro de la díada, y como resultados, se consideraron las conductas con que correspondió su cónyuge, con un retraso de 10 segundos (lag=1).

Dado que el sistema observacional incluye categorías conductuales que 
favorecen el intercambio social y también que lo limitan, éstas fueron clasificadas en conductas positivas y negativas respectivamente, en consonancia con lo propuesto por Adams (1965) con respecto a la cualidad de las contribuciones y valencia de los resultados.

Por lo tanto, las categorías de Verbalización acerca del tema, Habla Simultánea, Aprobación, Emisión, Interacción Física, Humor y Contacto Visual, se consideraron como contribuciones positivas, y las categorías de Desacuerdo, Defensa, Provocación, Dominante, Otras Conductas y Pérdida de Contacto Visual, se consideraron como contribuciones negativas. La misma distinción se aplica a los resultados, con la salvedad de que se presentan por el otro cónyuge de la díada con un retraso de 10 segundos.

De esta manera, las bases de datos originales se recategorizaron y se realizaron análisis secuenciales con lag= 1 en dos sentidos, con la conducta del marido como antecedente y la conducta de la esposa como consecuente, y posteriormente en el sentido inverso. De los análisis secuenciales se obtuvo cada elemento presente en el modelo teórico expresado en la segunda (modelo positivo) y tercera (modelo negativo) ecuación, con ellos se calcularon las razones relativas correspondientes y se solicitaron análisis de regresión entre las razones relativas de contribuciones y resultados. Tanto los análisis secuenciales como el análisis de regresión se realizaron con los datos de la interacción durante la discusión completa, y por secciones de discusión de manera independiente.

\section{Resultados.}

Los datos descriptivos de la información conductual se resumen en la Figura 1 , la cual muestra el promedio de frecuencia en la presentación de intervalos por hora de las categorías observacionales del SOIC. En el panel izquierdo de la Figura 1 se incluye la información correspondiente a las categorías de alta tasa: V, O, CV y X; y en el panel derecho se presentan las categorías de baja tasa: A, Hs, Emisión, D, Df, P, Do, F y H (ver figura 1).

Ambos grupos difieren significativamente en la categoría de Emisión: $t(1,20)=2.284 ; p=.037$, y presentan diferencias marginales en las categorías de Pérdida de Contacto Visual $(X): t(1,20)=2.077, p=.057$; y Humor $(H)$ : $t(1,20)=-2.019, p=.057$. El grupo de cónyuges violentos presenta mayor promedio de intervalos en Emisión y Pérdida de Contacto Visual, así como menor promedio de intervalos en Humor, que los controles.

La operación del modelo, se basó en datos de la secuencia conductual de la interacción social entre cónyuges, para lograr distinguir la interacción conyugal conflictiva de la que no lo es. Por lo tanto, a partir de análisis secuenciales se operaron la segunda y tercera ecuaciones, cuyos resultados se muestran en las siguientes figuras. 

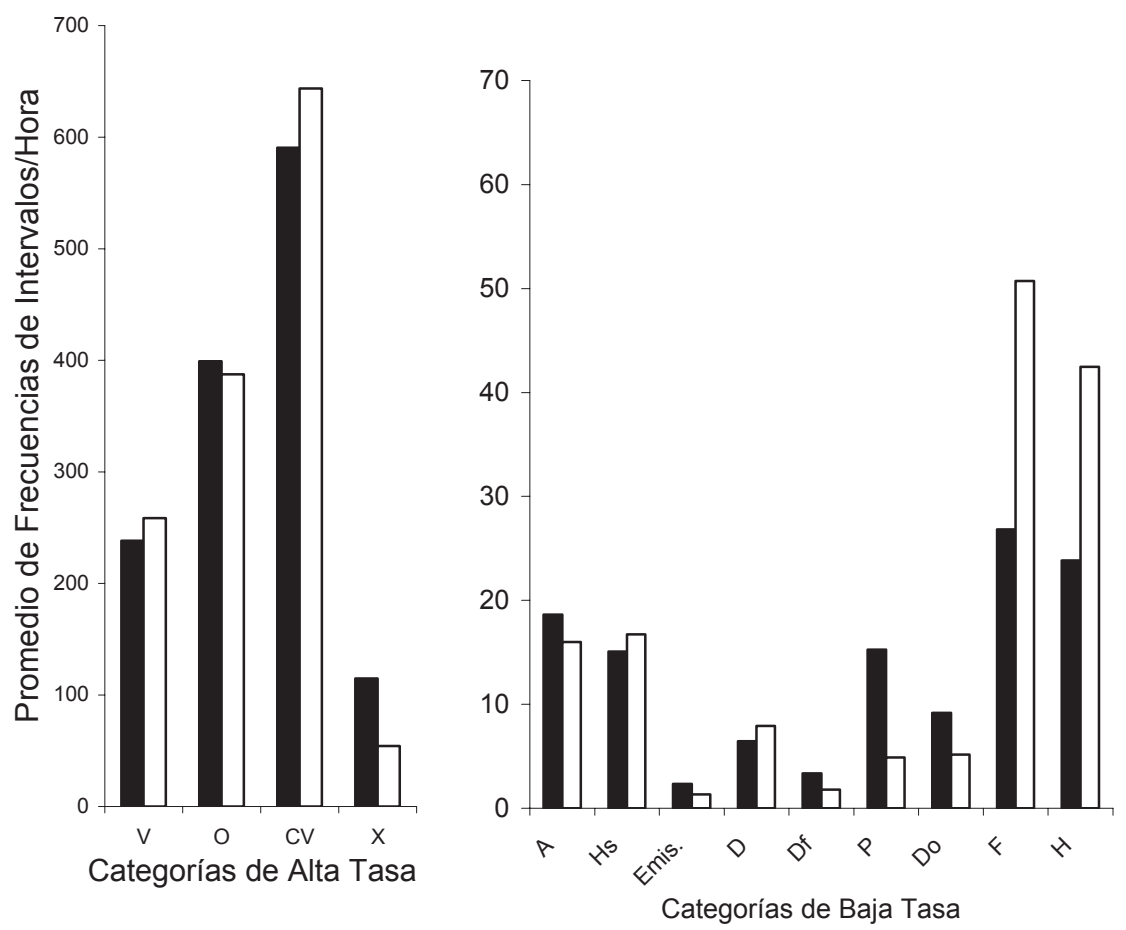

Grupo Violento

$\square$ Grupo de Control

Figura 1. Frecuencias Absolutas de Intervalos de Categorías del SOIC, de alta y de baja tasa, para Cónyuges Violentos y de Control. Se indica el promedio de intervalos/hora.

En las figuras se indican las frecuencias relativas de resultados y contribuciones del modelo, en que datos cercanos a uno implican mayores puntajes para el marido y menores puntajes para la esposa. Datos cercanos a cero implican menores puntajes para el marido y mayores puntajes para la esposa. En ambos casos, se definen condiciones de desequilibrio o asimetría. Datos cercanos a .5, implican puntajes similares para ambos cónyuges, e indica el equilibrio o simetría de la frecuencia relativa correspondiente.

La Figura 2 presenta las frecuencias relativas de contribuciones y resultados del modelo positivo durante la discusión completa, que es representativa de los datos obtenidos en las tres secciones de discusión. El panel izquier- 
do incluye los datos del grupo de cónyuges violentos, y el panel derecho, del grupo de control. Con la figura se evidencia que los datos de cónyuges violentos se distribuyen denotando una relación directamente proporcional, mientras que los datos del grupo de control se concentran alrededor del .5 en la escala de ambos ejes. Con lo anterior se demuestra que cónyuges violentos experimentan mayores niveles de asimetría en su interacción a diferencia del grupo de control, quienes evidencian ser más simétricos en sus intercambios conductuales.

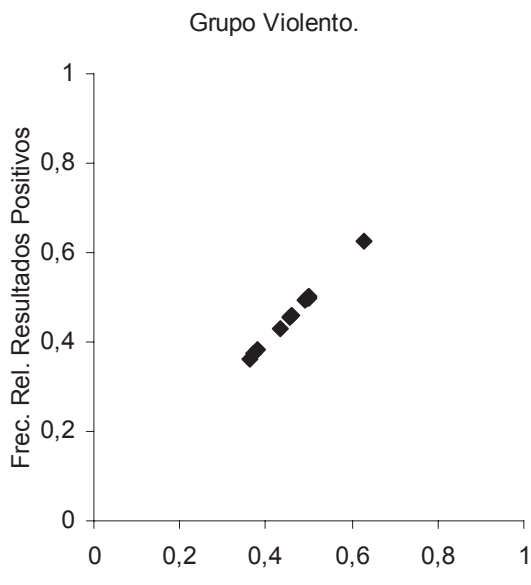

Frec. Rel. Contribuciones Positivas
Grupo de Control.

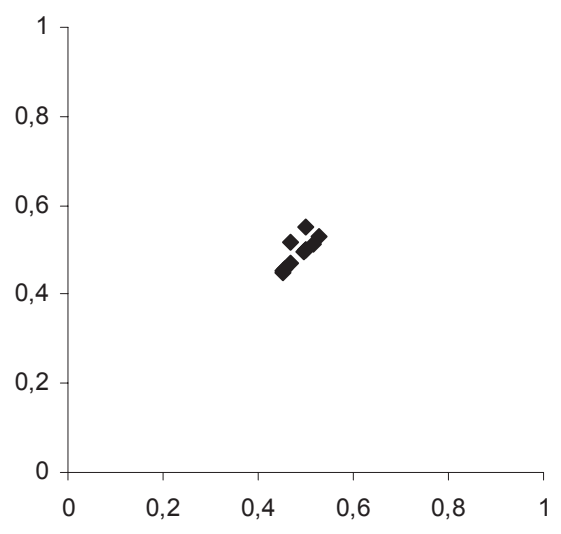

Frec. Rel. Contribuciones Positivas

Figura 2. Asimetría del modelo positivo. Frecuencias relativas de Contribuciones y Resultados. Cónyuges Violentos (panel izquierdo) y de Control (panel derecho). Discusión completa. Datos secuenciales con lag=1.

Dada la relación directamente proporcional observada en el panel izquierdo, se muestra que cónyuges violentos evidencian un patrón de incremento en sus frecuencias relativas de contribuciones y resultados positivos. Tal relación no está presente en el grupo de control. Los resultados anteriores se respaldan con el estadístico de prueba del análisis de regresión que presenta los siguientes valores para el grupo violento: intercepto: -.0025, pendiente: 1.006, error estándar: .003; Beta: 1.0; $p \leq .000$; coeficiente de determinación: 1.0; y para el grupo de control: intercepto: .176, pendiente: .625, error estándar: .164; Beta: .785; $p \leq .004$; coeficiente de determinación: .617. En estos valores se aprecia que el intercepto para el grupo violento es cercano a cero y el valor de la pendiente cercano a uno, características que definen matemáticamente una relación asimétrica y directamente proporcional, como se anticipó. En el 
grupo de control, estos valores son sensiblemente diferentes, implicando con ello que la relación establecida difiere de la anteriormente explicada.

Estas diferencias entre grupos están presentes en cada sección de discusión temática, lo que demuestra un patrón consistente en los intercambios sociales de cada grupo. Con fines de brevedad, las figuras correspondientes a los intercambios durante cada sección de discusión se obvian y los resultados estadísticos se resumen en la sección A de la Tabla 1 . En general, estos datos muestran que tanto los patrones de interacción social de las díadas violentas como los de las parejas control acopladas fueron adecuadamente descritos por los modelos propuestos (ecuaciones 2 y 3 ) bajo las tres situaciones de discusión.

Tabla 1. Análisis de Regresión de Contribuciones y Resultados del modelo positivo (sección A) y negativo (sección B). Se indican valores de $R^{2}$, intercepto, pendiente, error estándar, Beta y significancia de la prueba, por secciones de discusión de grupo violento y de control.

\begin{tabular}{|l|c|c|c|c|c|c|}
\hline Sección A & \multicolumn{5}{|c|}{ Modelo Positivo } \\
\hline & Grupo Violento. & & & & & \\
\hline & $\begin{array}{l}\text { Coeficiente de } \\
\text { Determinación }\end{array}$ & Intercepto & Pendiente & $\begin{array}{c}\text { Error } \\
\text { estándar }\end{array}$ & Beta & Sig. \\
\hline Evento Cotidiano & 1 & -.003046 & 1.008 & .007 & 1 & .00 \\
\hline Res. de Conflicto & .999 & -.00008131 & 0.999 & .008 & 1 & .00 \\
\hline Tóp. Placentero & .999 & -.004174 & 1.01 & .01 & 1 & .00 \\
\hline & Grupo Control & & & & & \\
\hline & $\begin{array}{l}\text { Coeficiente de } \\
\text { Determinación }\end{array}$ & Intercepto & Pendiente & $\begin{array}{c}\text { Error } \\
\text { estándar }\end{array}$ & Beta & Sig. \\
\hline Evento Cotidiano & .995 & -.004735 & 1.009 & .023 & .998 & .00 \\
\hline Res. de Conflicto & .999 & .004047 & 0.992 & .008 & 1 & .00 \\
\hline Tóp. Placentero & .933 & -.0954 & 1.2 & .107 & .966 & .00 \\
\hline Sección B & \multicolumn{2}{|c|}{ Modelo Negativo } & & & & \\
\hline & Grupo Violento. & \multicolumn{1}{|c|}{} & & & & \\
\hline & $\begin{array}{l}\text { Coeficiente de } \\
\text { Determinación }\end{array}$ & Intercepto & Pendiente & $\begin{array}{c}\text { Error } \\
\text { estándar }\end{array}$ & Beta & Sig. \\
\hline Evento Cotidiano & .999 & -.01143 & 1.019 & .008 & 1 & .00 \\
\hline Res. de Conflicto & .999 & .002581 & .997 & .009 & 1 & .00 \\
\hline Tóp. Placentero & .999 & -.0003156 & .999 & .01 & 1 & .00 \\
\hline & Grupo Control & & & & & \\
\hline & $\begin{array}{l}\text { Coeficiente de } \\
\text { Determinación }\end{array}$ & Intercepto & Pendiente & $\begin{array}{c}\text { Error } \\
\text { estándar }\end{array}$ & Beta & Sig. \\
\hline Evento Cotidiano & .994 & -.01028 & 1.021 & .026 & .997 & .00 \\
\hline Res. de Conflicto & .999 & .007002 & 0.985 & .008 & 1 & .00 \\
\hline Tóp. Placentero & .713 & -.239 & 1.453 & .307 & .845 & .01 \\
\hline
\end{tabular}


Los datos correspondientes al modelo negativo, se presentan en la Figura 3, que incluye los intercambios sociales durante la discusión completa y es representativa de los datos obtenidos durante cada sección de discusión. La información del grupo de cónyuges violentos se incluye en el panel izquierdo y la información para el grupo de control, en el derecho.

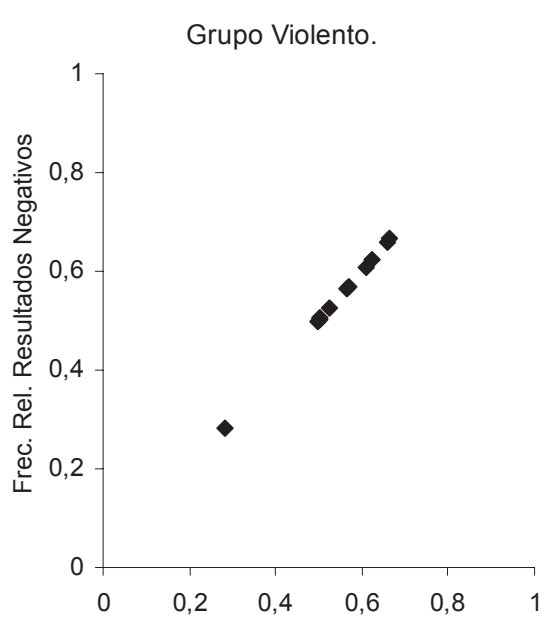

Frec. Rel. Contribuciones Negativas

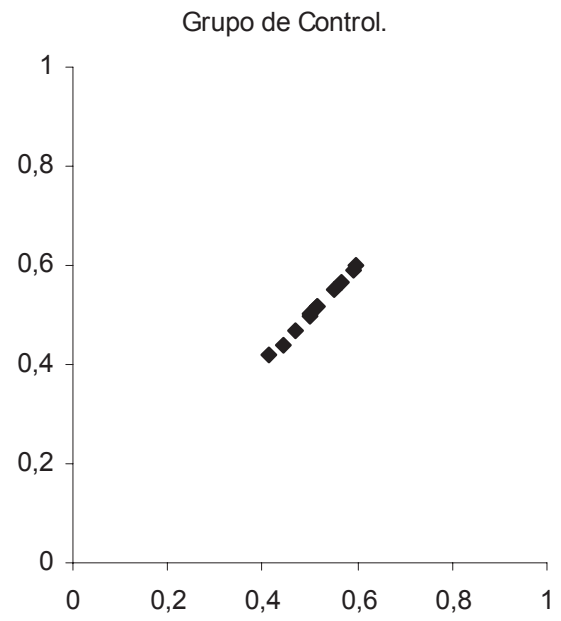

Frec. Rel. Contribuciones Negativas

Figura 3. Asimetría del modelo negativo. Frecuencias relativas de Contribuciones y Resultados. Cónyuges Violentos (panel izquierdo) y de Control (panel derecho). Discusión completa. Datos secuenciales con lag=1.

En esta figura se aprecia que para ambos grupos los puntos reflejan seguir una relación directamente proporcional, respaldada por los valores estadísticos de intercepto: -.001503, pendiente: 1.002, error estándar: .004; Beta: 1.0; $p \leq .000$; coeficiente de determinación: 1.0 para el grupo violento; y para el grupo de control, intercepto: -.003361, pendiente: 1.006, error estándar: .008; Beta: 1.0; $p \leq .000$; coeficiente de determinación: .999 para el grupo de control. Aunque el modelo ajusta bien en ambos grupos, la línea de puntos de cada grupo difiere en la dispersión en torno a los ejes. Para el grupo violento, los puntos se distribuyen desde el . 3 hasta el .7 en ambos ejes, mientras que los datos para el grupo de control se concentran entre el .4 y .6. Siendo más dispersos los datos del grupo violento, con ello se comprueba nuevamente que existen mayores niveles de asimetría en los intercambios sociales negativos entre cónyuges violentos que entre cónyuges de control. 
Los análisis por sección de discusión temática, evidencian la misma relación que en la discusión completa. Datos del grupo violento son más dispersos en ambos ejes que el grupo de control, por lo que las diferencias en el patrón de interacción entre ambos grupos es consistente. En este caso, al igual que en el modelo positivo, se abrevia la información gráfica de los intercambios durante secciones de discusión y la información estadística correspondiente se resume en la sección B de la Tabla 1.

En este punto surge la inquietud por determinar cuál es la naturaleza del mecanismo que opera en la interacción conyugal de ambos grupos y qué los hace diferentes, para lo cual el análisis secuencial sin retardo ofrece información al respecto, y complementa la prueba del modelo al identificar diferencias en la sincronía conductual de ambos grupos. Con este propósito, las bases de datos fueron arregladas para permitir análisis secuenciales con lag $=0$, empleando la recategorización de conductas explicada en el análisis anterior.

Los análisis secuenciales proporcionaron cuatro diferentes tipos de asociaciones de categorías: positivo-positivo, positivo-negativo, negativo-positivo y negativo-negativo. Lo anterior indica qué tipo de conductas presentan los cónyuges simultáneamente. Esto permite deducir que dos tipos de combinaciones (positivo-positivo y negativo-negativo) refieren estados de comportamiento sincrónicos. Con esta información se compararon ambos grupos en el promedio de frecuencia de presentación de cada combinación de categorías a través de una prueba $\mathrm{T}$, con los datos de la discusión completa y por sección de discusión de manera independiente. La Figura 4 presenta gráficamente la información descriptiva de los intercambios durante la discusión del Evento Cotidiano, en que se detectaron diferencias significativas entre grupos.

La Figura 4 muestra que la sincronía positiva presenta diferencias significativas entre grupos, siendo mayor el promedio para el grupo de control que para el grupo violento: $t(1,20)=2.143, p=.045$. También se presentan diferencias entre grupos en la sincronía negativa, que aunque marginalmente significativas, se incluyen por considerarse conceptualmente relevantes. En ella, el grupo violento presenta mayor promedio que el grupo de control: $\mathrm{t}(1,20)=2.027, \mathrm{p}=.056$, como se anticipó.

En conclusión, las díadas de control presentan mayor sincronía en categorías que favorecen el intercambio social (positivas), mientras que parejas violentas presentan mayor sincronía en las categorías que lo limitan (negativas). Las combinaciones positivo-negativo y negativo-positivo, no presentaron diferencias significativas.

Hasta aquí, los dos tipos de análisis reportados demuestran la sensibilidad del modelo asumido y el mecanismo diferencial implícito en la interacción, sin embargo, dada la generalidad del modelo, incorporada en el agrupamiento de conductas y de díadas, es necesario que se complemente el estudio de la interacción con información más detallada y fina, lo que es factible a 
través de un análisis microsocial (Patterson, 1982). El análisis secuencial por categorías individuales satisface este requerimiento.

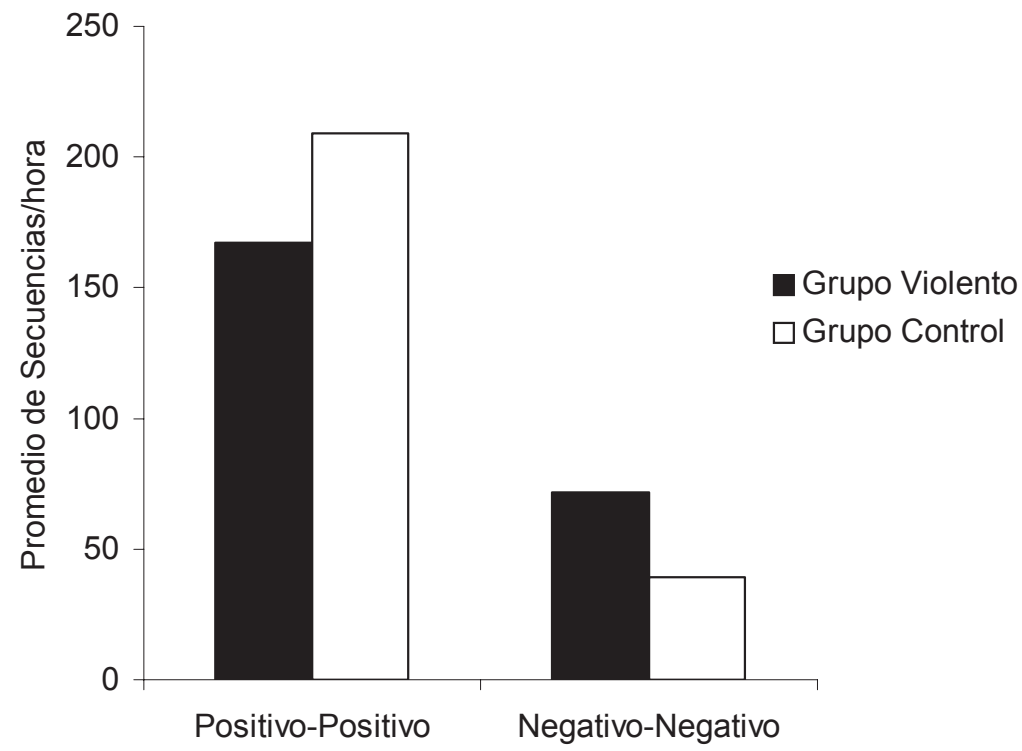

Sincronía Conductual lag $=0$

Figura 4. Sincronía conductual para grupo violento y de control durante la discusión del Evento Cotidiano. Se indica el promedio de secuencias/hora positivo-positivo y negativo-negativo. Datos secuenciales con lag $=0$.

A manera de ejemplo y con fines de brevedad, en las Figuras 5 y 6 se presenta la información sólo de una díada violenta y su control acoplado, respecto al patrón conductual excitatorio e inhibitorio, respectivamente. En ellas se resume la información obtenida de análisis secuenciales con lag=1 en dos sentidos, primero considerando como antecedente la conducta del marido y como consecuente la conducta de la esposa y posteriormente en el sentido inverso. En ambas figuras, formas rectangulares representan conductas emitidas por el marido, y formas redondeadas refieren a las presentadas por la esposa. Categorías positivas se representan con figuras claras y se sombrean aquellas categorías consideradas como negativas, de acuerdo a la categorización descrita en los análisis previos. Conectores de líneas punteadas representan probabilidades condicionales menores a .25 ; conectores 
de líneas sólidas delgadas refieren probabilidades condicionales iguales 0 mayores a .25 y menores de .5; conectores de líneas sólidas gruesas representan probabilidades condicionales iguales o mayores a .5 y menores de .75 ; y los conectores de mayor grosor corresponden a probabilidades condicionales iguales o superiores a .75 .

La Figura 5 indica las probabilidades condicionales entre categorías individuales cuyos residuales ajustados estandarizados fueron superiores a +1.96, de acuerdo con los criterios propuestos por Haberman (1978). Lo que significa que la frecuencia con que una conducta es consecuente de otra es superior a la que se esperaría por azar, con una probabilidad de error menor o igual a .05. Ello implica que una conducta es excitatoria de otra.

Dado que la prueba del modelo indica que cónyuges violentos presentan una relación directamente proporcional en sus frecuencias relativas de contribuciones y resultados para ambos modelos, positivo y negativo, el análisis secuencial permite especificar con mayor detalle cuáles categorías individuales se encuentran involucradas en dicha relación, y adicionalmente informa sobre la fuerza de la relación entre categorías, así como la secuencia conductual de ambos cónyuges

En la Figura 5 se incluye el diagrama de transición de la díada violenta y su control. En la díada violenta se pueden apreciar tres ciclos de secuencias conductuales. Dos de ellos ubicados a cada extremo de la figura, involucran categorías positivas, salvo la categoría de Otras Conductas, de marido y esposa respectivamente. El ciclo central involucra categorías negativas. En ambos tipos de secuencias resalta la bidireccionalidad de la relación entre cónyuges, es decir, conductas positivas del marido son correspondidas por categorías del mismo tipo de la esposa. Esta relación también se encuentra presente en las secuencias de categorías negativas, lo que indica que esta díada se ocupa en mayores secuencias negativas, ya que un cónyuge corresponde la conducta negativa del otro, con conducta del mismo tipo.

Esta tendencia de corresponder con categorías del mismo tipo no se encuentra presente en la díada de control, salvo en un caso, pero la relación es unidireccional, lo cual explica que cónyuges de control ocupen menos tiempo en secuencias de negatividad.

Además, se aprecia que la díada violenta presenta probabilidades incondicionales de categorías negativas mayores que la pareja control, y ésta por su parte, presenta probabilidades incondicionales superiores en categorías positivas respecto a la primera. La suma de las probabilidades incondicionales de la díada violenta es igual a la unidad, a diferencia de la díada de control, para la cual no fue necesario graficar todas sus categorías observacionales, ya que algunas de ellas no presentaron valores significativos en los análisis de residuales.

De lo anterior se deduce que la díada violenta presenta un más amplio 
patrón excitatorio entre conductas positivas y negativas, respectivamente, ya que se observa mayor variedad en las secuencias conductuales positivas y negativas, con lo que se confirma la relación directamente proporcional detectada para cónyuges violentos (anteriormente explicada) en el modelo positivo y negativo.

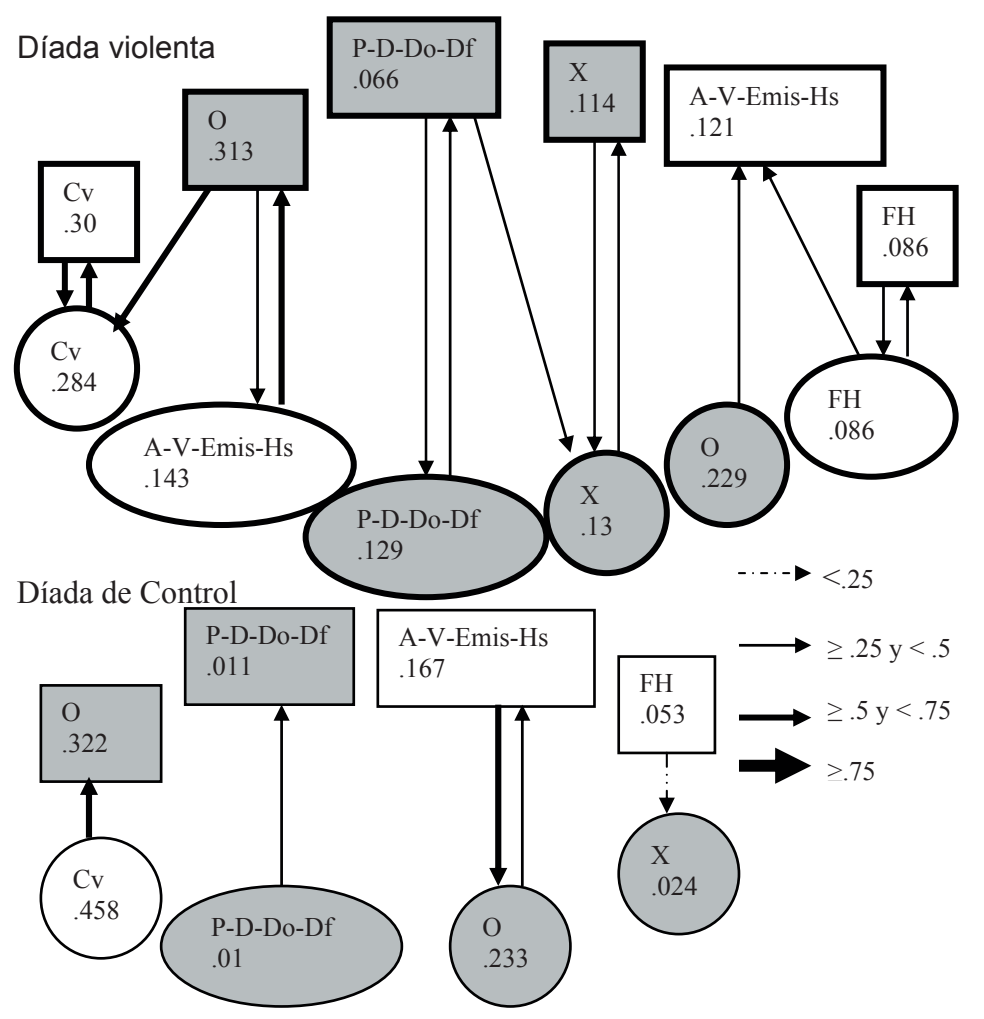

$\mathrm{A}=$ Aprobación; $\mathrm{V}=$ Verbalización acerca del tema; Emis=Emisión; $\mathrm{Hs}=$ Habla simultánea; D=Desacuerdo; Df=Defensa; Do=Dominante; $\mathrm{P}=$ Provocación;

$\mathrm{F}=$ Contacto Físico; $\mathrm{H}=\mathrm{Humor} ; \mathrm{Cv}=$ Contacto Visual; $\mathrm{X}=$ Pérdida de Contacto

Visual; $\mathrm{O}=$ Otras Conductas

Figura 5. Patrón excitatorio conductual para una díada violenta y control. Probabilidades incondicionales por categorías se incluyen dentro de los círculos o cuadros. Se indican solo probabilidades condicionales superiores a +1.96 de residuales ajustados estandarizados. 
En la Figura 6 se presentan las relaciones significativas encontradas a partir de los residuales ajustados estandarizados (superiores a -1.96), lo que indica que la ausencia de una conducta es consecuencia de la presentación de otra, superior a la que se esperaría por el azar, con una probabilidad de error menor o igual a .05. Por lo que este análisis proporciona información sobre relaciones inhibitorias entre categorías conductuales que exhiben ambos tipos de díadas.

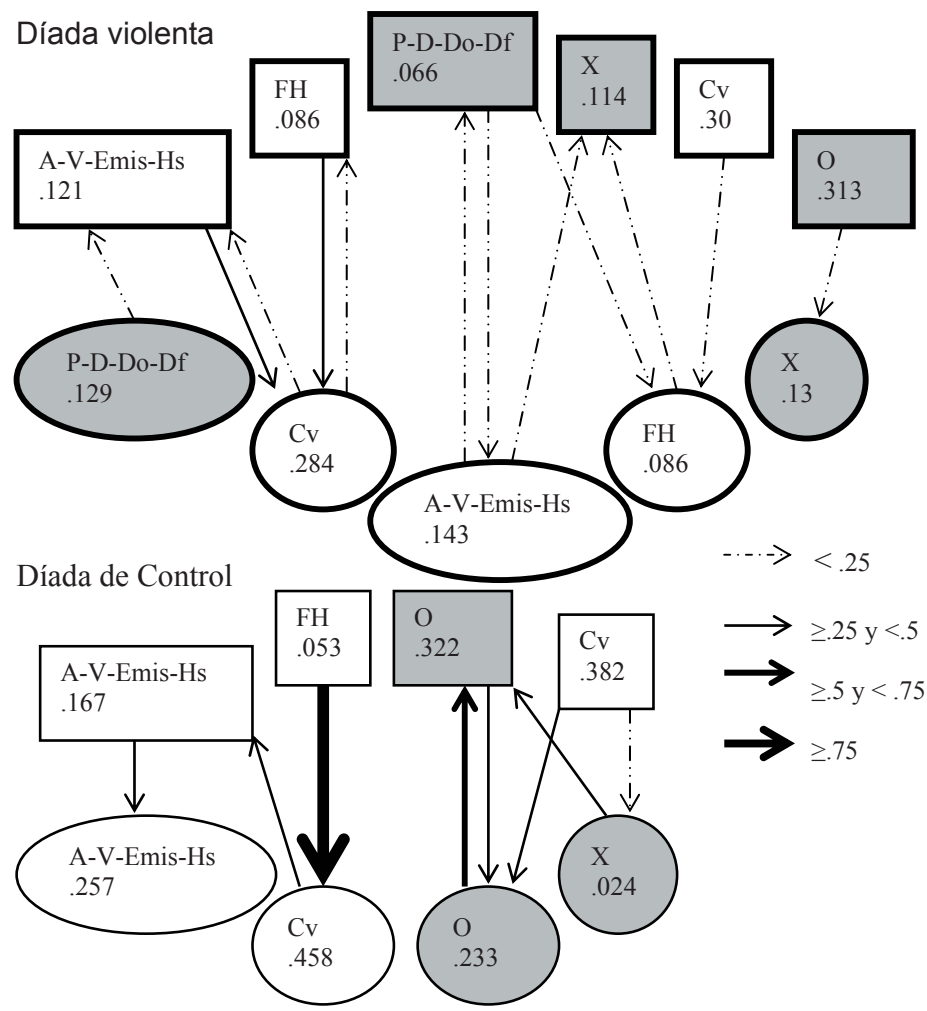

A=Aprobación; V=Verbalización acerca del tema; Emis=Emisión; Hs= Habla simultánea; $\mathrm{D}=$ Desacuerdo; $\mathrm{Df}=$ Defensa; $\mathrm{Do}=$ Dominante; $\mathrm{P}=$ Provocación; $\mathrm{F}=$ Contacto Físico; $\mathrm{H}=\mathrm{Humor} ; \mathrm{Cv}=$ Contacto Visual; $\mathrm{X}=$ Pérdida de Contacto Visual; $\mathrm{O}=$ Otras Conductas

Figura 6. Patrón inhibitorio conductual para una díada violenta y control. Se incluyen probabilidades incondicionales por categorías y se indican probabilidades condicionales superiores a -1.96 de residuales ajustados estandarizados. 
En la díada violenta se observa que la mayor parte de las probabilidades condicionales son débiles, con ello se implica que el poder inhibitorio conductual entre ambos cónyuges tiende a ser de bajo nivel. Por el contrario, la díada de control presenta probabilidades condicionales moderadas o fuertes (salvo en una secuencia), lo que significa que cónyuges de control son más efectivos al inhibir la conducta de su contraparte, esto explica en buena medida que cónyuges de control se ocupen en secuencias conductuales positivas y negativas de menor duración y sean más hábiles para salir de ciclos de negatividad.

Finalmente, se aprecia que conductas negativas de la díada violenta inhiben conductas positivas de su cónyuge de manera bidireccional, a diferencia de la pareja de control, en la cual se perciben ciclos inhibitorios de conductas negativas de manera bidireccional, y ciclos inhibitorios unidireccionales de conductas positivas.

De la misma manera que en la Figura 5 , la suma de las probabilidades incondicionales para la díada violenta es igual a la unidad, no así, para la díada de control, ya que ésta no presentó relaciones significativas en el análisis de residuales ajustados para algunas de sus categorías observacionales.

Discusión.

Los resultados de este estudio conducen a reconocer a los modelos de proporcionalidad (Adams, 1965; Anderson, 1991; Farkas, 1991), como válidos, útiles y fértiles a la investigación sobre el intercambio social entre cónyuges, incorporando al análisis la simetría y sincronía conductual. La principal contribución de este trabajo consistió en extender estos modelos al ámbito conductual, mismos que han sido útiles para describir la injusticia en comparaciones interpersonales a partir de los juicios de injusticia de los participantes. Con ello pueden iniciarse líneas de investigación en donde las propiedades de los episodios que realmente enfrentan los cónyuges son incorporados al análisis y no solo sus juicios o percepciones.

En esencia, el modelo de Farkas (1991) tomado como base, ha permitido fundamentar una estrategia de análisis de las secuencias conductuales para describir, distinguir y predecir los procesos de intercambio social que experimentan dos tipos de parejas, bajo diferentes situaciones de intercambios, con lo que se demuestra la amplia generalidad y valor predictivo del modelo. Esta estrategia basada en medidas directas de la interacción, proporciona amplio soporte al estudio de estos intercambios sociales, que la convierte en la base de un modelo capaz de resaltar relaciones que permanecerían imperceptibles a otros modelos y estrategias de análisis. Con ello se demuestra la fortaleza teórica y conceptual del modelo, y por otra parte, se subraya la necesidad de que la investigación en el área cuente con información obtenida de fuentes 
directas. Con este trabajo se demuestra que en el estudio de la interacción conyugal, es de fundamental importancia la naturaleza fina y detallada de los datos, ya que permite analizar secuencias conductuales y revelar relaciones que permanecen ocultas al análisis general e indiferenciado de la conducta, para lo cual, la Metodología Observacional resulta prometedora.

Al aplicar este modelo al ámbito conyugal, se demuestra que las díadas establecen coacciones, que parecen desapercibidas a la simple observación, y que pueden ser interpretadas como contribuciones y resultados de la relación. En este sentido, el estudio contribuye significativamente a extender el margen conceptual y generalidad de la Teoría de Integración de la Información (Anderson, 1991), puesto que en él se integra información de diversos dominios, como lo es, por una parte, conducta de maridos y esposas, y por otra, contribuciones y resultados de la relación.

Los resultados detectados con el estudio concuerdan con lo esperado y coinciden con la literatura, al determinar que existe mayor reciprocidad de afecto negativo en parejas infelices (Gottman, 1998); que relaciones inequitativas o asimétricas se relacionan con agresión marital (Leonard \& Senchak, 1996); que es necesario considerar el sentido de equidad o de justicia que el individuo establece en sus comparaciones interpersonales, para entender el crimen (Wilson \& Herrnstein, 1985), y que parejas angustiadas demuestran mayor asimetría en su interacción conyugal. Aunque en trabajos previos (Gottman, 1979) el concepto de asimetría haya sido manejado como la predictibilidad de la conducta de uno a partir del otro. Esto conduce a afirmar que el concepto de asimetría extiende su generalidad, dadas las diferentes operacionalizaciones y definiciones del término manejadas en la literatura.

Además, con estos hallazgos, se confirma que la violencia puede desempeñar un papel funcional para restablecer la equidad diádica, y reafirma la idea acerca de que en el área del matrimonio opera un balance conductual entre negatividad y positividad, idea fundamental para la teoría conductual del balance en el matrimonio, referida en algunos estudios relevantes (Gottman, 1993), que coincide con lo establecido por Adams (1965), en torno a la cualidad de las contribuciones y valencia de los resultados, distinción que constituyó un elemento clave en el análisis del presente estudio.

Adicionalmente, con el estudio se especifican aquellos mecanismos diferenciales que constituyen patrones excitatorios e inhibitorios conductuales de parejas violentas y de control, que complementan el análisis del modelo teórico, y que coinciden con los referidos en la literatura (Patterson, 1982; Gottman, 1979). De ellos se deduce la implicación práctica de estos resultados para programas de prevención y tratamiento de la violencia conyugal y consejería marital.

Consideramos que un análisis más fino del sistema observacional de la interacción conyugal, permitirá encontrar diferencias más contundentes en 
el patrón de sincronía conductual entre cónyuges, y determinar así, que la sincronía para intercambios negativos y positivos de cónyuges violentos y de control, respectivamente, se evidencie en los intercambios de toda la discusión y no solamente en una sección de ella. Esta necesidad de mayor fineza en el análisis de la interacción conyugal, complementada con análisis de los contenidos abordados en la discusión y de medidas fisiológicas, representan áreas de oportunidad para investigación posterior, en coherencia con lo señalado por Gottman y Notarius (2002), respecto a preferir aproximaciones multimétodo en el abordaje de los procesos bajo estudio.

\section{Referencias}

Adams, J. S. (1965). Inequity in social exchange. En L. Berkowitz (Ed.) Advances in Experimental Social Psychology (vol. 2, pp. 267-299), New York: Academic Press.

Anderson, N. H. (Ed.). (1991). Contributions to information integration theory. Cognition (Vol. 1), Hillsdale, N. J.: Lawrence Erlbaum Ass.

Anglin, K. \& Holtzworth-Munroe, A. (1997). Comparing the responses or maritally violent and nonviolent spouses to problematic marital and nonmarital situations: Are the skill deficits of physically aggressive husbands and wives global?. Journal of Family Psychology, 11 (3), 301-313.

Babcock, J. C., Waltz, J., Jacobson, N. S. \& Gottman, J. M. (1993). Power and violence. The relation between communication patterns, power discrepancies and domestic violence. Journal of Consulting and Clinical Psychology, 61 (1), 40-50.

Bakeman, R. \& Gottman, J. M. (1989). Observación de la interacción: introducción al análisis secuencial. (M. T. Anguera \& A. Blanco, Trads.). Madrid: Morata. (Trabajo original publicado en 1986).

Carstensen, L. L., Gottman, J. M. \& Levenson, R. W. (1995). Emotional behavior in long-term marriage. Psychology and Aging, 10 (1), 140-149.

Cascardi, M. \& Vivian, D. (1995). Context for specific episodes of marital violence: Gender and severity of violence differences. Journal of Family Violence, 10 (3), 265-293.

Ehrensaft, M. K. \& Vivian, D. (1996). Spouses' reasons for not reporting existing marital aggression as a marital problem. Journal of Family Psychology, 10 (4), 443-453.

Farkas, A. J. (1991). Cognitive algebra of interpersonal unfairness. En N. H. Anderson (Ed.), Contributions to Information Integration Theory. Cognition (vol. 1, pp. 4399). Hillsdale, N. J: Lawrence Erlbaum Associates.

Gottman, J. M. (1979). Marital Interaction. Experimental Investigations. New York: Academic Press.

Gottman, J. M. (1993). The roles of conflict engagement, escalation, and avoidance in marital interaction: A longitudinal view of five types of couples. Journal of Consulting and Clinical Psychology, 61 (1), 6-15.

Gottman, J. M. (1998). Psychology and the study of marital processes. Annual Review of Psychology, 49, 169-197. 
Gottman, J. M. \& Notarius, I. (2002). Marital research in the 20th century and a research agenda for the 21st century. Family Process, 41(2), 139-197.

Haberman, S. J. (1978). Analysis of qualitative data. Vol. 1: Introductory topics. New York: Academic Press.

Heyman, R. E., O’Leary, K. D. \& Jouriles, E. N. (1995). Alcohol and aggressive personality styles: Potentiators of serious physical aggression against wives? Journal of Family Psychology, 9 (1), 44-57.

Holtzworth-Munroe, A., Markman, H., O'Leary, K. \& Neidig, P. (1995). The need for marital violence prevention efforts: A behavioral-cognitive secondary prevention program for engaged and newly married couples. Applied and Preventive Psychology, 4 (2), 77-88.

Langhinrichsen-Rohling, J., Neidig, P. \& Thorn, G. (1995). Violent marriages: Gender differences in levels of current violence and past abuse. Journal of Family Violence, 10 (2), 159-176.

Leonard, K. E. \& Senchak, M. (1996). Prospective prediction of husband marital aggression within newlywed couples. Journal of Abnormal Psychology, 105 (3), 369-380

Levenson, R. W., Carstensen, L. L. \& Gottman, J. M. (1994). The influence of age and gender on affect, physiology, and their interrelations. A study of long-term marriages. Journal of Personality and Social Psychology, 67 (1), 56-68.

López, C. E. \& Santoyo, V. C. (2001, julio). Desarrollo de un sistema observacional de codificación de la interacción conyugal. En C. Santoyo V. (Coordinador), Aportaciones de la metodología observacional al análisis de la conducta. Simposio efectuado en el XV Congreso de la Sociedad Mexicana de Análisis de la Conducta, Toluca, Edo. de México.

Margolin, G. (1987). The multiple forms of aggressiveness between marital partners: How do we identify them?. Journal of Marital and Family Therapy, 13 (1), 77-84.

O’Leary, K. D., Malone, J. \& Tyree, A. (1994). Physical aggression in early marriage: Prerelationship and relationship effects. Journal of Consulting and Clinical Psychology, 62 (3), 594-602.

Patterson, G. R. (1982). Coercive family processes. A social learning approach (Vol. 3), Eugene OR: Castlia.

Potegal, M. \& Knutson, J. F. (Eds.). (1994). The dynamics of aggression: Biological and social processes in dyads and groups. New Jersey: Lawrence Erlbaum Associates.

Quera, J. V. (1991). Muestreo y registro observacional. En M. T. Anguera (Ed.). Metodología observacional en la investigación psicológica (vol. 1, pp. 241-327), Barcelona: Promociones y Publicaciones Universitarias.

Stroebe, W., Stroebe, M., Abakoumkin, G. \& Schut. H. (1996). The role of loneliness and social support in adjustment to loss: A test of attachment versus stress theory. Journal of Personality and Social Psychology, 70 (6), 1241-1249.

Sugarman, D. B., Aldarongo, E. \& Boney M. S. (1996). Risk marker analysis of husband-to-wife violence: A continuum of aggression. Journal of Applied Social Psychology, 26 ( 4), 313-337.

Vivian, D. \& Langhinrichsen-Rohling, J. (1994). Are bi-directionally violent couples mutually victimized? A gender-sensitive comparison. Violence and Victims, 9 (2), 107-124. 
Widom, C. S. (1989). Does violence beget violence?: A critical examination of the literature. Psychological bulletin, 106 (1), 3-28.

Wilson, J. Q. \& Herrnstein, R. J. (1985). Crime and Human Nature. New York: Simon and Schuster.

\section{Apéndice 1. Sistema Observacional de la Interacción Conyugal.}

\begin{tabular}{|c|c|c|c|}
\hline \multicolumn{2}{|c|}{ Categorías/Subcategorías } & Definición & Código \\
\hline \multicolumn{2}{|c|}{$\begin{array}{l}\text { Verbalización acerca del } \\
\text { tema. }\end{array}$} & $\begin{array}{l}\text { Hacer una declaración verbal que comunica } \\
\text { información relacionada con la tarea solicitada } \\
\text { cuando los participantes esperan turnos. }\end{array}$ & V \\
\hline \multicolumn{2}{|c|}{ Habla simultánea } & $\begin{array}{l}\text { Hacer una declaración verbal al mismo tiempo que } \\
\text { el otro. Se distingue de verbalización acerca del } \\
\text { tema, en que no se esperan turnos. }\end{array}$ & $\mathrm{Hs}$ \\
\hline Aprobación & $\begin{array}{l}{ }^{*} \text { Asentir } \\
\text { *Reforzar } \\
\text { verbalmente }\end{array}$ & $\begin{array}{l}\text { *Expresiones no verbales que reflejan acuerdo } \\
\text { o aprobación hacia el otro, en ausencia de } \\
\text { verbalización. } \\
\text { ^Expresiones verbales que impliquen aceptación, } \\
\text { admiración y/o valoración como consecuencia de } \\
\text { conductas realizadas por el otro. }\end{array}$ & $A$ \\
\hline Desacuerdo. & $\begin{array}{l}\text { *Expresar } \\
\text { Desacuerdo } \\
\text { *Negar } \\
\\
\text { *Levantar los } \\
\text { ojos sin mover } \\
\text { la cabeza }\end{array}$ & $\begin{array}{l}\text { *Expresiones verbales que denotan desaprobación } \\
\text { hacia la conducta o características de la otra } \\
\text { persona } \\
\text { *Mover horizontalmente la cabeza, siempre y } \\
\text { cuando no se utilice para enfatizar lo que se afirma } \\
\text { verbalmente. } \\
\text { *Dirigir la mirada hacia arriba sin mover la cabeza, } \\
\text { de manera que la pupila se oculta parcialmente en el } \\
\text { párpado superior. }\end{array}$ & D \\
\hline Defensa & $\begin{array}{l}\text { *Excusarse } \\
\text { *Ignorar } \\
\text { *Respuestas } \\
\text { asociadas al } \\
\text { llanto }\end{array}$ & $\begin{array}{l}\text { *Incrementar la distancia física del otro: voltear la } \\
\text { cabeza o cuerpo en dirección opuesta del cónyuge, } \\
\text { cuando no lleva el objetivo de encontrar comodidad } \\
\text { física o postural. } \\
\text { *Explicación verbal acerca de una conducta o una } \\
\text { actividad realizada por el sujeto, cuando el otro } \\
\text { manifiesta una crítica, queja o reclamo. } \\
\text { ^Realizar una conducta incompatible a la conducta } \\
\text { del otro, por ejemplo: hablar por teléfono, levantarse, } \\
\text { salirse del escenario. } \\
\text { *Dirigir hacia abajo las comisuras de los labios y } \\
\text { apretar los músculos de las mejillas, en ocasiones } \\
\text { asociada con producción de lágrimas. }\end{array}$ & Df \\
\hline
\end{tabular}




\begin{tabular}{|c|c|c|c|}
\hline \multicolumn{2}{|c|}{ Categorías/Subcategorías } & \multirow[b]{2}{*}{\begin{tabular}{|l} 
Definición \\
*Hacer comentarios embarazosos o avergonzantes \\
de la otra persona, incluye el reírse del otro. \\
*Señalar características de la conducta o actividades \\
realizadas por el otro implicando valoración \\
de incomodidad o inconformidad. Se incluyen \\
declaraciones irónicas, de reclamo y queja. \\
*Declaración verbal que expresa admiración por la \\
conducta o características del otro, realizada con \\
una expresión facial y tono de voz incoherentes con \\
lo verbalmente expresado.
\end{tabular}} & \multirow{2}{*}{\begin{tabular}{|l} 
Código \\
P
\end{tabular}} \\
\hline Provocación & ${ }^{*}$ Burlarse & & \\
\hline Dominante & *Amenazar & $\begin{array}{l}\text { *Dar al otro la instrucción de hacer algo o de no } \\
\text { hacerlo, implicando el cumplimiento inmediato de la } \\
\text { misma. } \\
\text { *Anticipar eventos negativos o desagradables para } \\
\text { el otro. } \\
\text { *Hacer una declaración verbal al mismo tiempo } \\
\text { que el otro, cuando alguno incrementa su voz } \\
\text { en "staccato", y ninguno de los dos participantes } \\
\text { detiene su conducta. }\end{array}$ & Do \\
\hline \multicolumn{2}{|l|}{ Emisión } & $\begin{array}{l}\text { Indica al sujeto que inicia la interacción de alguna de } \\
\text { las tres áreas de discusión. }\end{array}$ & Emis \\
\hline \multicolumn{2}{|c|}{ Otras Conductas } & $\begin{array}{l}\text { Pausas en el flujo de conducta del sujeto no } \\
\text { consideradas en las demás categorías. P.ej. mirar } \\
\text { hacia otras partes de la habitación, tocar su propio } \\
\text { cuerpo, bostezar, manipulación de objetos, en } \\
\text { ausencia del resto de conductas. }\end{array}$ & 0 \\
\hline \multicolumn{2}{|c|}{ Interacción Física } & $\begin{array}{l}\text { Buscar y/o mantener cercanía física con el otro, que } \\
\text { incluye tocar, besar y/o acariciar. }\end{array}$ & $\mathrm{F}$ \\
\hline Humor & *Sonreír. & $\begin{array}{l}\text { *Expresión de la parte inferior de la cara que } \\
\text { consiste en una elevación evidente de las comisuras } \\
\text { de los labios haciendo una emisión sonora. } \\
\text { *Expresión en la parte inferior de la cara que } \\
\text { consiste en una ligera elevación de las comisuras de } \\
\text { los labios, sin emisión sonora. }\end{array}$ & $\mathrm{H}$ \\
\hline \multicolumn{2}{|c|}{ Contacto Visual } & $\begin{array}{l}\text { Enfocar la mirada hacia el otro. Incluye mirar el } \\
\text { cuerpo del otro a un ángulo no mayor de } 90 \text { grados } \\
\text { vertical u horizontalmente. }\end{array}$ & Cv \\
\hline \multicolumn{2}{|c|}{ Pérdida de Contacto Visual } & $\begin{array}{l}\text { Enfocar la mirada lejos del otro. Incluye mirar en } \\
\text { dirección distinta de cualquier parte del cuerpo del } \\
\text { otro, en un ángulo mayor de } 90 \text { grados vertical u } \\
\text { horizontalmente. }\end{array}$ & $x$ \\
\hline
\end{tabular}

